\title{
Tolerancia y tendencias racionalistas en la primera Europa moderna
}

\author{
MIQUEL BELTRÁN \\ Instituto de Filosofia (CSIC)
}

\begin{abstract}
RESUMEN
La cuestión de la libertad de conciencia, en los Paises Bajos, tomó forma en torno a la tolerancia que desplegaban las sectas más moderadas de protestantes - pero también los dirigentes politicos-con respecto a los refugiados que iban llegando a tierras holandesas procedentes de lugares donde la libertad de religión se hallaba seriamente amenazada. Así ocurrió con los judíos que. procedentes de España y Portugal, Ilegaron a Amsierdam a finales del siglo XVI, y también con los socinianos, seeta que tuvo su origen en Polonia y que, entre otras cosas, negaba la divinidad de Cristo. Se pretende probar en el artículo que fueron, con todo, motivaciones económicas las que se hallaban en la base de la peculiar tolerancia que se manifestó en los Países Bajos durante el XVII -asi lo prueban los escritos de los hermanos De la Court, y observaciones de extranjeros de la talla de William Temple, entre otros muchos documentos-.
\end{abstract}

$$
\text { PAL.ABRAS CL.AVE }
$$

TOLPRANCIA-UJBERTAD DE CONCIENCIA-SECTAS REUIOOSAS-ECONOMIA

ABSTRACT

In the Netherlands, the issue of freedom of conscience was advanced by the practice of toleration adopted by moderate protestant sects - and likewise by political leaders-towards the refugees coming from places where religious freedom was seriously threatened. That was the case of the Spanish and Portuguese Jews who arrived in Amsterdam at the end of the XVIth century. An equal fate had the Socinians, a Polish seet that, aomeng other things, denied the divinity of Christ. The paper argues that economic reasons were at the base of hte peculiar toleration experienced in the Netherlands along the XVIIth century. The point is proved by the writings of the De la Court brothers, as well as by evidences of foreigners like William Temple, among many other documents.

KEYWOROS

TOLERATION-FREEEOM OF CONSCIENCE-RELIGIOUS SECTS-ECONOMY 
En una Carta dirigida al polaco Jean Stornski, firmada el 7 de Agosto de 1638, Grocio (Ep. 1006) escribía: «Libertas sentiendi quae velis et dicendi quae sentias legibus Regni apud vos nicitur». Esta tolerancia polaca frente al establecimiento de otras religiones, que pareció ejemplar a los extranjeros, como la de Holanda se lo pareció también a muchos de los que la contemplaban desde fuera en la segunda mitad del XVII, era en efecto insólita para un país católico. Y en efecto, los protestantes polacos tenían conciencia de gozar de mejor suerte que sus correligionarios en cualquier otro país en que la religión romana fuese mayoritaria. Sin embargo, la condición de convivencia entre religiones distintas variaba en Polonia de una ciudad a otra. Los luteranos, que tenían el poder en las ciudades de la Prusia Real, excluían sistemáticamente a los católicos de los cargos municipales y de las corporaciones, y ciertas ciudades católicas procedían de igual modo con los protestantes.

Con todo, las grandes ciudades reales eran liberales, y la política sobre este extremo expresada por el príncipe Etienne Bathory pareció determinante en dicha dirección, al profesar éste públicamente su convicción de que la libertad religiosa era un bien en sí mismo, y de que Dios se había reservado para sí estas tres cosas: el crear algo de la nada, el conocer lo que ha de venir, y el gobernar las conciencias. Bathory declaró asimismo que él era rey de los pueblos y no de las conciencias, $y$ aunque sus intervenciones políticas efectivas en materia de religión se alejaron bastante de esta profesión, un pacto de tolerancia parecía planear sobre las reclamaciones de los protestantes que pedían una ley especial para la represión de los tumultos en religión. En este orden de cosas Fausto Socino fue llamado a Transilvania para combatir ciertas doctrinas heterodoxas. Allí, gracias a sus polémicas contra los impíos, Socino se ganó la confianza de los Hermanos Polacos - cuya célebre Biblioteca Fratrum Polonorum fue publicada, precisamente en Holanda, en la segunda mitad del XVII- y se encargó de persuadirles de los problemas teológicos que conllevaba el anabaptismo y la adoración de Cristo.

Poco a poco Socino los inclinó a aceptar la teología que había estado elaborando ya desde antes de refugiarse en Polonia, y en la cual había trabajado durante años con el fin de lograr una exposición de la misma clara y sistemática para que sus discípulos, tras su muerte, pudieran editarla. Dicha doctrina, sobre la que se basa el Catecismo de Raków, está fundada sobre la negación del pecado original: el pecado original no existe y por lo tanto no puede en absoluto viciar el libre albedrío humano, de modo que el hombre es perfectamente libre, y Cristo, en consecuencia, no ha venido al mundo a rescatarlo, al menos en calidad de Dios. Socino llama a Cristo servator o conservator, esto es, guardián o protector, y no salvator o redemptor. Cristo sólo ha venido a revelar a los hombres el camino de la vida eterna y así Socino profesó -al igual que algunos heréticos de Padua como Camilo Renato y otros antitrinitarios 
italianos- la creencia de que el alma es mortal como el cuerpo, porque el hombre, por naturaleza, nada tiene en común con la inmortalidad. Por la misma razón de que, desde el origen, fue creado a partir de un puñado de tierra, el hombre ha sido creado mortal.

Lo importante aquí es la reconsideración del papel de Cristo que la negación del pecado original supone, y que permite entender la confesión de fe antitrinitaria: Cristo intercede ante Dios por nosotros y nos salvaguarda del pecado por el poder que Dios le ha concedido, pero simplemente como hombre. Además, esta gracia sobre los hombres la distribuye al margen de cualquier sacramento. Los sacramentos son meras ceremonias rituales, y la eucaristía una más entre ellas, establecida, eso sí, por Cristo en la práctica. No es difícil imaginar que a este culto sin sacramentos le hace falta tan sólo una iglesia no muy organizada. Yen realidad el Catecismo de Raków parece confudir la iglesia visible con la iglesia invisible. En el catecismo se lee que no sirve de gran cosa buscar los signos de la verdadera iglesia de Cristo, porque toda asamblea que crea y profese la doctrina de la salvación es la verdadera iglesia de Cristo, y aquel que abraze la doctrina de la salvación está ya en la verdadera iglesia. Así Socino, en una carta a Radecke del 24 de Septiembre de 1584, escribía que consideraba como verdaderas iglesias todas aquellas en las que resuena la voz de Cristo, y donde se obedezca a esta voz, incluso si le pareciera que su opinión sobre ciertos dogmas no es la correcta, o que en ellas no se atiende apropiadamente a los mandamientos. Aún más, Socino consideró que la creencia en la Trinidad era un error lamentable, pero que no comprometía irremediablemente la salvación.

El Catecismo de Raków fue impreso por primera vez en 1605, y esta publicación, como afirmó Pintacuda de Michelis (1975, pp. 11-12) «rappresenta il momento dell'avvenuta e completa omogeneizzazione dottrinale ed ecclesiastica tra gruppi di credenti in dissidio con luterani e riformati sul tema della trinità, sensibili alle suggestioni dell'anabattismo, fortemente impegnati nella realizzazione pratica dei precetti cristiani». Así, la fisonomía moral y doctrinal de las iglesias antitrinitarias y disidentes en Polonia durante el XVI encuentra una definición clara en el Catecismo. Tras una primera edición latina en 1609, la evolución doctrinal del socinianismo condujo a una pronta necesidad de revisión del texto, revisión que fue encargada por el sínodo de Raków a Johannes Crell. Crell sería el autor de un texto fundamental en la evolución de la doctrina de la religión natural en el XVII, el Liber de Deo et ejus attributis, de 1630. Pero mientras Crell se dedicaba a la revisión del catecismo, el advenimiento al poder en Polonia de la dinastía Wasa se concretó en una mayor intolerancia, condicionada sobre todo por el avance de la contrareforma católica en el país. La política de intoleracia practicada por esos reyes se cebó especialmente en los socinianos y culminó con su expulsión del país, tras una serie de 
represiones como la clausura de locales de culto socinianos, o el hecho de excluirles de las garantías religiosas que se habían asegurado a los disidentes en la Confederación de Varsovia del 1573, pero sobre todo con la destrucción del centro de Raców, en particular la escuela y la tipografía, en 1638.

Después de estos eventos los viajes por Europa que los socinianos estaban realizando para difundir su mensaje se intensificaron, transformándose en una verdadera diáspora. Y en 1658 salió a la luz un decreto que convertía en proscritos a todos los socinianos de Polonia en el margen de dos años, $\mathrm{y}$ al cual se dio una rígida vigencia a partir de 1660 .

El movimiento sociniano pudo reagruparse y adquirió cierta fuerza en Amsterdam, debido a la peculiar tolerancia que se practicaba en los Países Bajos con los miembros de otras religiones, pero fue sobre todo el hecho de que allí se había producido un movimiento de disidencia con respecto a la principal ortodoxia protestante, movimiento -el de los colegiantes- en cuyo interior la inclusión de los antitrinitarios no fue, dados los presupuestos de la agrupación terriblemente problemática, lo que hizo posible esa reagrupación de los socinianos.

El movimiento colegiante había nacido como consecuencia de la dispersión de los arminianos tras la derrota política que éstos sufrieron frente a los gomaristas en el sínodo de Dordrecht (1618-19). En éste, y pese a la preeminencia intelectual de los arminianos, llamados también remostrantes, se produjo la previsible aniquilación de los mismos, debida al apoyo político que sus adversarios habían conseguido de parte del estatúder Maurice de Nassau. Así, los ministros de la facción vencida fueron depuestos, y considerados enemigos de la patria, tuvieron que decidir entre exiliarse del país o sufrir prisión (Grocio fue uno de los hombre encarcelados en aras de la polémica), aunque a algunos de los pastores remostrantes políticamente irrelevantes se les permitió la permanencia en Holanda con la condición de que no predicaran más. Fix explica de qué modo la dispersión que siguió al sínodo tuvo una de sus consecuencias en la aparición de los colegiantes: «Entre las congregaciones remostrantes que se quedaron sin dirigente en 1620 , debido al exilio forzado de los pastores arminianos, estaba la congregación de la diminuta villa de Warmond cerca de Leiden. Antes que aceptar a un ministro gomarista o dispersarse por falta de dirigente, la congregación decidió seguir los consejos de uno de sus mayores...y continuar agrupándose sin predicador. Los miembros de la congregación se reunían para orar en común, cantar himnos y leer la Biblia en voz alta, basándose para la interpretación bíblica y la educación religiosa en el testimonio espontáneo, inspirado, de todo miembro del grupo que se sintiese movido por el Espíritu Santo para hablar por mor del esclarecimiento del grupo. Esta práctica, conocida como 'libre profecía', llegó a ser el rasgo característico de un nuevo movimiento religioso dedicado a la religión moral y espiritual, altamen- 
te crítico con respecto a la Iglesia reformada doctrinaria, institucionalizada. El colegio, como llegaron a ser llamados esos encuentros, continuó reuniéndose en secreto (por temor a la persecución calvinista) en Warmond durante 1620 antes de trasladarse a la vecina villa de Rijnsburg en 1621. Había nacido el movimiento colegiante» (Fix 1987, p. 68).

Los colegiantes empezaron siendo milenaristas y espiritualistas, influidos como estaban por la reforma radical del XVI y por el arminianismo en Holan$\mathrm{da}$, pero progresivamente pasaron a ser un grupo de pensadores racionalistas que atravesaron un período de religión racional antes de llegar al racionalismo filosófico secularizado que encontró su expresión culminante en Spinoza (quien estuvo, como se sabe, en estrecho contacto con colegiantes como Balling y Jelles ya desde antes de su expulsión de la sinagoga). Fix (1991) ha examinado el complejo proceso de transformación intelectual de los colegiantes y también sus raíces culturales y religiosas. Los años de desarrollo del pensamiento colegiante fueron también los del proceso por el cual la fe en una providencia divina especial, y la creencia en la operación directa de la voluntad divina sobre el mundo, empezaron a ser reemplazadas por un punto de vista secularizado que emplazaba la presencia divina fuera del ámbito de decisión humano, y de la actividad natural en general. El reto a la autoridad eclesiástica tradicional que supuso la Reforma estaba en el origen de esa transición de la fe a la razón, y también de las teorías de la resistencia en los siglos XVI y XVII, $y$ así en el del nacimiento de la tolerancia moderna, $y$ tal como yo he intentado probar en otro trabajo (Beltrán, 1993), la acepción según la cual tolerancia consiste en soportar pacientemente el punto de vista religioso de otro está en la base de la utilización de la palabra 'verdaagzawheid' en la Holanda del XVII, aunque como Huseman (1984, p. 301) ha demostrado, la tolerancia de doctrinas contrarias en religión fue claramente percibida en primer lugar como una paciente aceptación de algo negativo («tolérer takes only negative objects»), a lo largo del siglo XVI.

Los racionalistas holandeses creyeron que la conciencia individual que operaba a través de la razón humana natural era una fuente de verdad tan infalible en sus resultados como la inspiración divina lo fue para los primeros espiritualistas. Pero esta creencia, madurada por los colegiantes a lo largo de décadas, tuvo en parte su inicio en el descreimiento de que Dios operaba directamente sobre las acciones del hombre, y este abandono, a su vez, fue producto en parte del desencanto que causó el conflictivo curso tomado por la reforma protestante, y las terribles masacres de las guerras de religión, desde la noche de San Bartolomé hasta la guerra de los treinta años, que convencieron a muchos de que Dios ya no podía estar directamente implicado en los asuntos humanos. La rivalidad entre iglesias y la presunta incompatibilidad de algunos de sus presupuestos de base llevaron a hacer pensar que no había posibilidad 
en absoluto de llegar a la verdad en materia de religión. Para muchos el mundo se convirtió en un ámbito para la actividad del hombre de la cual ni la inspiración ni la revelación divinas formaban parte. Los colegiantes fueron uno de esos grupos que llegó a pensar -casi por eliminación-que la sola razón era la única guía que quedaba para la verdad y para la acción justa. Así, esta vía hacia la secularización y el racionalismo se desarrolló a partir de la desesperación religiosa. Ello se produjo sobre todo en la segunda mitad del XVII, y especialmente en Inglaterra, donde algunos hombres de iglesia anglicanos percibieron la necesidad de supeditar la autoridad a la razón en materia de religión. Estos liberales anglicanos propusieron una iglesia comprehensiva y desarrollaron la doctrina de la adiáspora: la idea de que ciertas doctrinas y rituales podían no ser considerados esenciales para la fe cristiana. Sobre este presupuesto, la diversidad de opinión debía ser perfectamente tolerada.

$\mathrm{Y}$ aunque la diversidad de creencias religiosas condujera con presteza al deísmo y a la secularización, los teólogos protestantes que le dieron origen pretendían diversamente situar a la cristiandad sobre una base racional con el fin de derrotar los argumentos de ateístas, calvinistas ortodoxos y católicos. Así, latitudinarios como John Tillotson o John Wilkins creían que la razón humana podía aprehender lo esencial de la religión sin necesidad alguna de recurrir a la autoridad externa, pero creían también que la religión natural precisaba para su establecimiento de verse complementada por la revelación.

La transformación hacia lo secular en el pensamiento religioso que aconteció en Holanda, de efectos portentosos, tuvo también causas excepcionales: ante todo, la implicación entre comercio y tolerancia que se dio en los Países Bajos con una suerte de reciprocidad, y ello a pesar de que la situación política y geográfica de los Países Bajos era en principio extraordinariamente adversa para que se produjera su prosperidad económica, porque todo parecía obrar en contra de dicha posibilidad. Los Países Bajos llegaron a su esplendor en el marco de una organización política poco menos que medieval. Ya en 1576, dos años antes de que los primeros pastores calvinistas hicieran su irrupción en las Provincias Unidas, siete estados habían acordado enviar delegados a unos Estados Generales que se constituyeron nuevamente entonces. Holanda, el estado más importante de entre aquellos, tenía por lo demás una complicada estructura gubernamental. Su principal cuerpo legislativo, los Estados de Holan$\mathrm{da}$, se hallaba constituido por 18 representantes de las distintas ciudades, y por un representante de la nobleza en general. Tampoco había monarca en las Provincias Unidas. Lo más próximo a ello era el cargo de estatúder, que desempenaron los príncipes de Orange, pero que sin embargo implicaba meramente ejercer el papel de un funcionario provincial. Los de Orange eran normalmente estatúders de varias provincias, aunque no de todas simultáneamente. Parece difícil, me parece, imaginar una estructura en apariencia menos pertinente para 
un desarrollo económico eficaz. Y sin embargo fue en el interior de esta estructura política que se produjo el progreso mercantil de Holanda, gracias quizá al contrapunto político que, frente a la figura del estatúder, comportaba la de otro funcionario administrativo provincial, que era el abogado del país. Se trataba del pensionario general, conocido como Gran Pensionario por los extranjeros, que se convirtió en la práctica, como afirma Wallerstein (1980), en el primer ministro de las Provincias Unidas, actuando incluso como presidente durante los dos períodos en los que no hubo estatúders.

Pero la aparente desorganización política era tan sólo uno de los problemas de principio para que se produjese la hegemonía de Holanda en la economía-mundo. Puesto que el suelo geográfico de los Países Bajos no era propicio para el cultivo, no puede dudarse del acierto con que la mayoría de estudiosos de su auge económico razonan que fue la pesca el gran motor inicial del esplendor mercantil de las Provincias Unidas (sobre todo la del arenque, al que se llegó a llamar la mina de oro de Europa). Pero, como afirma Wallerstein (1980), 'el arenque no lo explica todo'. Los holandeses mostraron idéntica tenacidad y superioridad en la agricultura, lo que no dejó de ser un logro prodigioso, dadas las adversas condiciones del suelo. Y además, Holanda llegó a ser también un principal productor industrial, cuyo progreso fue destacado sobre todo en el sector textil. Lo importante sin embargo es que en el desarrollo de su economía los habitantes de los Países Bajos del Norte se dieron cuenta de que la afluencia de refugiados político-religiosos, iniciada ya en fecha tan temprana como 1560, representaba beneficios enormes para ellos. Y que éste fue verdaderamente el principal factor en la revolución holandesa. Una industria predominante a partir de entonces fue la producción de libros, porque la Amberes del siglo XVI, ciudad de la que procedían muchos de los refugiados referidos, era el centro de la industria tipográfica del orbe, arte cuyo saber esos exiliados transmitieron a Amsterdam. Se ha olvidado pues, con frecuencia, en el análisis económico de la hegemonía holandesa, la existencia de estos otros factores, en particular la relación causa-efecto entre el interés y la libertad religiosa, relación que Johan de la Court, el estadista holandés más notable en tiempos de Spinoza, no duda en establecer de esta suerte: si el comercio requiere libertad, la libertad de religión es el medio mejor para atraer y conservar a los extranjeros, sin los cuales las empresas de Holanda se verían obligadas a dar un sueldo tan elevado a sus obreros y criados, que éstos se llevarían una parte importante de los beneficios y vivirían mejor que sus amos ( $c f$. Consideratien van Staat ofte Politike Weegschaal, 1661). Parece claro, por lo anterior, que los holandeses entendieron como un nada despreciable interés económico la promoción de la tolerancia para con los miembros de otras religiones que llegaban a sus Provincias escapando del campo de batalla que era el resto de Europa, y que la clase comerciante tuvo que tener un gran empeño en impedir que los calvinistas 
-por decirlo con una feliz expresión de Boxer (1965)- 'sacrificaran la ganancia a la piedad'.

Sin embargo y como ya he apuntado, fue la represión de los calvinistas ortodoxos la que dio lugar, tras la derrota de los arminianos, a la aparición de los colegiantes. Como resultado de sus orígenes remostrantes, el movimiento colegiante incorporó en su pensamiento religioso las principales críticas arminianas a la Iglesia reformada, entre ellas el rechazo de la predestinación, del confesionalismo y de la rigidez doctrinal, todo ello en favor de una tolerancia religiosa y de un cristianismo moralmente afianzado. $\mathrm{Y}$ así, dada su oposición a la Iglesia reformada oficial, el movimiento colegiante actuó como un imán atrayendo a su seno a grupos de protestantes radicales que llegaron a Holanda durante el XVI y a lo largo del XVII. Hacia mitad de este último siglo el socinianismo había llegado a ser parte integrante del movimiento colegiante. Esta presencia se incrementó sobre todo en la década de los 60 a raíz del decreto de expulsión de los mismos dictado en Polonia al que ya he hecho referencia. Como he apuntado también, y aunque entre los primeros socinianos la discusión sobre la tolerancia religiosa no es un tema que se halle presente de forma autónoma y precisa, existe en sus textos la predisposición general hacia una actitud tolerante para con todos los cristianos, predisposición que se halla ya en Socino, y que surge de la preeminencia reservada al momento ético sobre el dogmático en la vida religiosa. Samuel Przypkowski, quizá el más renombrado de los antitrinitarios atacados por Leibniz, fue el primer sociniano al cual debemos una obra entera y exclusivamente dedicada al problema de la tolerancia religiosa. Desde 1611 a 1619 Przypkowki estuvo en la Universidad de Leiden, en un momento político y religioso crucial para los Paises Bajos, pues se trata de los años en los que se produjo el descalabro político de los arminianos. Henry Méchoulan (1990) ha explicado recientemente cómo una disputa en principio meramente teológica -la de Arminio y Gomaro en torno al dogma de la predestinación-pudo convertirse en un affaire político de primera magnitud y de repercusiones inusitadas. En medio de la querella, Przypkowski tuvo una relación directa con uno de sus mayores protagonistas, Episcopio, el teólogo que se encargó de defender a la parte arminiana en Dordrecht. Las posiciones de Przypkowski y Episcopio tenían en común una revalorización de la razón y de la voluntad humanas, consideradas como no esencialmente corruptas, y también la afirmación de la libertad del albedrío en un sentido por el cual se devuelve a la religión su momento práctico y la ética vuelve a constituir una parte esencial del cristianismo. En este sentido el deber de la tolerancia será de cumplimiento fundamental para el cristiano.

También en Johannes Crell, cuya más llamativa presencia en la cultura europea la representa su polémica contra una obra de Grocio que apareció en Leiden en 1617 con el título Defensio fidei Catholicae de satisfactione Christi 
adversus Faustum Socinum, escribió contra ésta una Responsio que se publicó en Raków en 1623. En ambas obras, sin embargo, el llamado a la tolerancia precede a la vindicación concreta de los dogmas, y así, frente a la exigencia de desarrollar un examen racional y profundo de la Sagrada Escritura y al sentimiento de la necesidad de que este examen se nutra de propósitos de paz frente a otros cristianos, y de justicia en el plano moral, las divergencias doctrinales específicas entre ambos disputantes -Grocio y Crell-pierden relieve y emerge por contra el sentido de una unidad de intención y una profunda solidaridad intelectual que halla su más alta expresión en lo que nos ha quedado de la correspondencia entre los dos hombres, que se produjo tras la publicación del libro de Crell. Crell escribió también, en torno a 1632, sus Vindiciae pro religionis libertate, en un momento en que para los socinianos la discusión sobre la tolerancia religiosa no representaba un problema relativo sólo a la coherencia de la profesión de fe cristiana, sino que estaba llegando a ser una cuestión de mera supervivencia. Con su De Deo et ejus attributis, Crell nos legó un tratado teológico orgánico sobre la esencia de Dios y sus atributos, de modo que puede decirse que nos hallamos ante el primer intento sociniano de construir una teología racional sistemática. Los sucesivos revisores del catecismo de Raków -entre ellos Crell, Ruar y Wistowaty-son los artífices del potenciamiento de los poderes de la razón con respecto al originario escrituralismo socianiano, pero la Religio rationalis (1685) de Andreas Wistowazy, ultimada en 1677, presenta los artículos de la religión natural no como deducciones racionales sino como 'axiomata quaedam universalia atque communes notiones', casi hasta esconder púdicamente el hecho que ahora la razón no se limitaba sólo a comprobar la no contradicción en las escrituras, sino que además permitía verificar su contenido a la luz de una teología construida independientemente del texto sacro.

Las obras de Pryzpkowski y de Crell hicieron que cuando los refugiados socinianos polacos llegaron en masse a las Provincias Unidas durante la década de los 60 encontraron en parte el terreno abonado para su integración en círculos remostrantes. Y aunque incluso las tolerantes autoridades holandesas fueron incapaces de asegurarles el derecho a ejercer su culto abiertamente, puesto que la iglesia ortodoxa ejercía una presión constante -aunque en su mayor parte infructuosa- sobre el gobierno civil, porque la historia del pensamiento libre en la república holandesa durante el XVII fue en gran medida la historia de una contienda constante entre el clericato calvinista y los regentes. Los primeros pedían vínculos más firmes entre Iglesia y Estado que permitieran un aumento del control clerical sobre los aspectos cotidianos de la convivencia entre religiones. Los regentes sin embargo no deseaban aumentar el poder político de la iglesia oficial, los socinianos fueron bien recibidos en los encuentros colegiantes donde se les permitió la libre expresión de sus ideas a través de la libre profecía. Esta atmósfera de tolerancia extrema prevalecía en 
los Colegios porque sus integrantes creían que dicha tolerancia era un aspecto de la espiritualidad prístina de la Iglesia primitiva que ellos aspiraban a hacer revivir. Nadie fue censurado por sus ideas en los encuentros colegiantes, e incluso el antitrinitarismo sociniano provocó escasa oposición. Ahora bien, aunque los socinianos no convirtieron a sus creencias a muchos colegiantes, algunas de sus ideas influyeron en el desarrollo del pensamiento de éstos, en particular el acercamiento racional a la interpretación bíblica, un acercamiento heredado por Socino del humanismo italiano.

A diferencia de las congregaciones de reformadores en el XVI, los colegiantes no creían estar en la exclusiva posesión de la inspiración y revelación divinas, y llegaron a considerar que en el mundo premilenario y corrupto de su época no había una única iglesia de Cristo. Para los primeros colegiantes el elemento esencial de la verdadera religión era la luz interior, la inspiración directa del Espíritu Santo sobre el alma del creyente individual, y como afirma Fix, nada en el devenir del movimiento colegiante ilustra más claramente la transición intelectual de la fe hasta la razón que los cambios en esa concepción de la luz interior. En su pensamiento los colegiantes combinaron el espiritualismo tradicional de la luz interior, heredado de Sebastian Frank y Gaspar Schwenckfeld, con las tendencias secularizadas presentes ya en un remostrante como Galenus Abrahamsz, quien popularizó entre sus correligionarios las críticas a la corrupta iglesia visible. La razón llegó paulatinamente a ser más que la mera y servil intérprete del contenido revelado de la verdad en religión, para convertirse por propio derecho en fuente de la verdad religiosa. Las obras de Balling y Jelles fueron importantes textos de transición entre el espiritualismo de colegiantes como Galenus y el racionalismo casi exclusivamente filósofico de Jan Bredenburg. El poder y la autoridad ganados por la razón humana identificada casi con la luz divina crearon el fundamento epistemológico para un sistema racional poderosamente argumentado. El contacto de Jelles y Balling con el cartesianismo, probablemente a través de Spinoza, les impulsó más aún en esa dirección, pero fue la influencia masiva de las ideas socinianas durante esos años la que acendró la tendencia racionalista en los colegiantes, aunque también la ferviente disputa doctrinal frente a las teorías de quákeros ingleses seguidores de William Ames, que polemizaron directamente con colegiantes como Serrarius, los inclinó a abandonar la creencia espiritualista en la luz interior.

El apogeo de esta tendencia lo constituye la Mathematica Demonstratio de Bredenburg, claramente influida por Spinoza, en la cual se define el ser o Ente necesario como aquel cuya existencia es su esencia, y aunque la obra se inicia con una vívida profesión de fe cristiana y con la súplica de Bredenburg para quienquiera que supiese indicarle el modo de hacer concordar religión y filosofía, 'con el fin de que su ánimo se viese restituido a la antigua tranquili- 
dad', éste se empeña en defender la idea de la verdad absoluta de la filosofía y también, a la vez, la de los contenidos especulativos de la escritura, ideas que según el autor no concuerdan sin embargo necesariamente entre sí, y por lo tanto el colegiante recurre a una suerte de doctrina de la doble verdad. Bredenburg había llegado así a la extrema conclusión de que las verdades de la razón y de la religión podían a menudo ser contradictorias, y que, a despecho de esta contradicción, debían mantenerse ambas, razón y revelación, como independientes, válidas y autónomas fuentes de verdad religiosa, de modo que se llegó en el movimiento colegiante a una concepción del conocimiento secular humano muy próxima a la separación completa entre filosofía y teología que Spinoza había propuesto en su Tractatus Theologico-Politicus, obra que el propio Bredenburg se había encargado en una primera época de intentar refutar.

\section{REFERENCIAS BIBLIOGRÁFICAS}

BELTRÁN, M. 1993: «Libertà di coscienza e tolleranza nell'opera di Spinoza», Rivista di Filosofia Neo-Scolastica, 86, pp. 738-746.

BOXER, C.R, 1965: The Dutch Seaborne Empire 1600-1800. New York: Knopf.

BREDENBURG, J. 1703, 1684: Winkunstige Demonstratie, trad. lat. Mathematica Demonstratio quod omnia entia, rationis capacia, necessario operantur. Amsterdam.

CRELL, J. 1630: Liber de Deo et ejus attributis. Racoviae. Reeditado en el vol. I de las Obras de Crell contenido en la Biblioteca Fratrum Polonorum. Irenopoli, post annum 1656 (1668).

DE LA COURT, J. y P. 1661: Consideratien van Staat ofte Politike Weegschaal. Amsterdam: J. Volckertsz Zinbreker.

FIX, A. C. 1987: «The Radical Reformation and Second Reformation in Holland. The Intellectual Consequences of the Sixteenth-Century Religious Upheaval and the coming of a Rational World View», The Sixteenth-Century Journal, XVIII, pp. 63-80.

1991: Prophecy and Reason: The Dutch Collegiants in the Early Enlightment. Princeton: Princeton University Press.

GROTIUS, H. 1679: Opera Omnia Theologica. Amstelaedami in folio, 4 vols.

HUSEMAN, W. H. 1984: «The expression of the Idea of Tolerance in French during the Sixteenth-Century», Sixteenth-Century Journal, XV, pp. 293-310.

JOBERT, A. 1974: De Luther à Mohila. La Pologne dans la Crise de la Chrétienté 1517-1648. Paris: Institute d'Etudes Slaves.

KOLAKOWSKI, L. 1969, 1965: Chrétiens sans Eglise. Traducción francesa del polaco, Paris: Gallimard.

LAGREE, J. 1991: La raison ardente. Religion naturelle et raison aux XVIIe siècle. Paris: Vrin.

MECHOULAN, H. 1990: Amsterdam au temps de Spinoza. Argent et Liberté. Paris PUF. 
NYE, S. 1687: A Brief History of the Unitarians, called also Socinians. In four Letters written to a Friend. Firmin.

PINTACUDA DE MICHELIS, F. 1975: Socinianesimo e tolleranza nell'età del razionalismo. Firenze: La Nuova Italia .

PRYZPKOWSKI, S. 1692: Cogitationes sacrae ad initium Evangelii Matthaei et omnes epistolas apostolicas nec non tractatus varii argumenti praecipue de iure Christiani Magistratus. Amsterdam: Eleutheropoli.

SCRIBANO, M. E. 1988: Da Descartes a Spinoza. Percorsi della teologia razionale nel Seicento. Milano: Franco Angeli.

SOZZINI, F.1656: Opera Omnia, 2 vols., en Biblioteca Fratrum Polonorum. Irenopoli, post annum domini 1656.

SPINOZA, B.1924: Spinoza Opera, ed. C. Gebhardt. Heidelbereg: Carl Winters.

WALLERSTEIN, I. 1980: The Modern World-System II. Mercantilism and the consolidation of the European World Economy 1600-1750, (traducción castellana de P. López, Madrid: Siglo XXI, 1984).

Miquel Beltrán trabaja como investigador en el Instituto de Filosofía del CSIC. Autor de «The God of the Tractatus Theologico-Politicus», North American Spinoza Society Monograph, 3, pp. 23-33, y «Spinoza: del elogio de Amsterdam a la epístola a Burgh», Isegoría, 14, pp. 164-173.

Dirección postal: Instituto de Filosofía (CSIC), Pinar 25, E-28006 Madrid. 